\title{
Ray trajectories of lower hybrid solitary structures
}

\author{
P. W. Schuck ${ }^{1,2}$ \\ Plasma Physics Division, Naval Research Laboratory, Washington, D.C., USA
}

\section{J. W. Bonnell}

Space Sciences Laboratory, University of California, Berkeley, California, USA

Received 2 October 2002; revised 25 November 2002; accepted 6 December 2002; published 8 May 2003.

[1] The correlation between large-amplitude lower hybrid waves and density depletions, termed "spikelets," "lower hybrid cavities," or "lower hybrid solitary structures" has been attributed to the change in the refractive index computed from the homogeneous plasma dispersion relation. However, the appropriate refractive index includes the electron Hall term involving the density gradient. We demonstrate that the Hall term produces ray trajectories which are profoundly different from the homogeneous plasma dispersion relation. INDEX TERMS: 2487 Ionosphere: Wave propagation (6934); 2471 Ionosphere: Plasma waves and instabilities; 2712 Magnetospheric Physics: Electric fields (2411); 2481 Ionosphere: Topside ionosphere; KEYWORDS: lower hybrid waves, solitary structures, ray-tracing, density depletions, Hall current, lower hybrid solitary structures

Citation: Schuck, P. W., and J. W. Bonnell, Ray trajectories of lower hybrid solitary structures, J. Geophys. Res., 108(A5), 1175, doi:10.1029/2002JA009716, 2003.

\section{Introduction}

[2] LHSS are intense regions of electric field fluctuations in isolated field-aligned cylindrical plasma density depletions at frequencies near the local lower hybrid resonance (LHR)

$$
\omega_{\mathrm{R}}^{2}(\mathbf{x}) \equiv \frac{\omega_{\mathrm{i}}^{2}(\mathbf{x}) \Omega_{\mathrm{e}}^{2}}{\omega_{\mathrm{e}}^{2}(\mathbf{x})+\Omega_{\mathrm{e}}^{2}},
$$

where $\omega_{j}(\mathbf{x}) \equiv e^{2} n(\mathbf{x}) / \epsilon_{0} m_{j}(j=\mathrm{i}, \mathrm{e})$ is the local species plasma frequency, $\Omega_{j}=e B_{0} / m_{j}$ is the cyclotron frequency, $m_{j}$ is the mass, $n(\mathbf{x})$ is the local plasma density, $B_{0}$ is the magnetic field strength, $e$ is the elementary charge, and $\epsilon_{0}$ is the permittivity of free space. This wave activity often exhibits significant spectral content from half to a few times the ambient LHR which is determined from the electrostatic cutoff in the spectrum outside of the density depletion [Brice and Smith, 1964, 1965].

[3] Lower hybrid solitary structures (LHSS), "spikelets," or lower hybrid cavities are a ubiquitous phenomenon in the auroral ionosphere [Labelle et al., 1986; Kintner et al., 1992; Vago et al., 1992; Ergun et al., 1995; Delory, 1996; Pinçon et al., 1997; Schuck et al., 1998; Bonnell et al., 1998; Delory et al., 1998; Knudsen et al., 1999; Dovner et al., 1994; Eriksson et al., 1994; Pécseli et al., 1996, 1997; Kjus et al., 1997, 1998; Høymork et al., 2000, 2001]. Indeed, Kjus et al. [1997] suggest that LHSS "represent the example of solitary wave structures to occur most abundantly in nature."

\footnotetext{
${ }^{1}$ Now at LET Corporation, Washington, D.C., USA.

${ }^{2}$ National Research Council Postdoctoral Fellow.

Copyright 2003 by the American Geophysical Union. 0148-0227/03/2002JA009716\$09.00
}

Certainly LHSS are observed by a majority of the sounding rockets launched into the auroral ionosphere during substorms [Labelle et al., 1986; Kintner et al., 1992; Delory, 1996; Pinçon et al., 1997; Schuck et al., 1998; Bonnell et al., 1998; Knudsen et al., 1999]. At higher altitudes, LHSS “can be found anywhere in regions where the Freja satellite has scanned, as long as ... lower hybrid waves of moderate amplitude" are present [Dovner et al., 1997, p. 622]. However, LHSS are usually observed at the boundaries of inverted-V events and just outside "active regions" of intense high energy particle precipitation [Lynch et al., 1994; Dovner et al., 1997; Kjus et al., 1997].

[4] Statistical analyses of Freja data between 1,100$1,750 \mathrm{~km}$ altitude demonstrates that the density profiles of LHSS are Gaussian and probably cylindrically shaped in the plane perpendicular to the geomagnetic field [Pécseli et al., 1996; Kjus et al., 1997, 1998; Høymork et al., 2000, 2001]. The cavity widths vary somewhat from orbit to orbit. However, in a detailed study of orbit 1234, Høymork et al. [2000] show that the mean $1 / e$-width of the cavities perpendicular to the magnetic field is about $30 \mathrm{~m}$ with a spread of $\pm 15 \mathrm{~m}$. The mean relative depth of the cavities is about $2 \%$ with observations rarely exceeding $10 \%$. LHSS at lower altitudes exhibit similar perpendicular scale sizes [Lynch et al., 1999], but the TOPAZ III sounding rocket detected considerably deeper striations occasionally approaching 80\% [Arnoldy et al., 1992; Kintner et al., 1992; Vago et al., 1992]. These observations were initially questioned by Ergun et al. [1994, 1995], but the OEDIPUS C sounding rocket observed $50 \%$ density depletions using two independent Langmuir probes: one in ion saturation and the other in electron saturation [Knudsen et al., 1999]. Furthermore, data from the PHAZE II sounding rocket exhibits small-scale plasma density variations of $50 \%$ 
inferred by tracking changes in the local plasma frequency [McAdams et al.,1998]. The extent of the density striations along the geomagnetic field has not been characterized but it is certainly several kilometers [Knudsen et al., 1999] and probably tens to hundreds of kilometers.

[5] Using the search coil magnetometer carried by the TOPAZ II sounding rocket, Vago et al. [1992] qualitatively demonstrates that LHSS are an essentially electrostatic phenomenon. Freja data [Dovner et al., 1994; Eriksson et al., 1994; Pécseli et al., 1996; Kjus et al., 1998; Høymork et al., 2000, 2001] consistently support this conclusion. Høymork et al. [2000] (p. 18,528) state that "Only very small magnetic fields are observed, and they show little or no correlation with the variations in electric field;" Dovner et al. [1994] report $E / B \gtrsim 8 \times 10^{7}(\mathrm{~m} / \mathrm{s})$ and Kjus et al. [1998] report $E / B \gtrsim 10^{6}(\mathrm{~m} / \mathrm{s})$. All three works conclude that these fluctuations are electrostatic. Since $E$ and $B$ are uncorrelated in these measurements, the value of $E / B \sim 10^{6}-10^{8} \mathrm{~m} / \mathrm{s}$ represents a lower bound for this ratio. Generally the raw $E / B$ ratio cannot be used exclusively to conclude that the fluctuations on the whistler-lower-hybrid branch are electrostatic particularly when this ratio is less than the speed of light $c \sim 3 \times 10^{8} \mathrm{~m} / \mathrm{s}$. However, Eriksson et al. [1994] presents spectra for LHSS where $E / B>7.5 c$ at frequencies above the ambient lower hybrid resonance. This last observation provides the most convincing quantitative evidence using the $E / B$ ratio that LHSS are electrostatic. Although the perpendicular scale length of the phenomena is perhaps a more rigorous criterion for concluding that fluctuations on the whistler-lower hybrid dispersion branch are in the electrostatic regime because the scale length is closely related to ratio of the longitudinal and transverse electric field components (P. W. Schuck et al., Properties of lower hybrid solitary structures: A comparison between space observations, a laboratory experiment, and the cold homogeneous plasma dispersion relation, submitted to Journal of Geophysical Research, 2003).

[6] By estimating the local frequency-wave number spectrum formulated using a Morlet wavelet basis, several authors demonstrate that the wave fronts in the cylindrical density striations rotate about center of the density striation [Pinçon et al., 1997; Schuck et al., 1998; Bonnell et al., 1998; Delory et al., 1998]. In particular, Bonnell et al. [1998] unambiguously demonstrate that LHSS are composed of right-hand(left-hand) rotating electric fields above(below) the ambient LHR where the "handedness" is determined relative to the direction of the geomagnetic field. At a minimum, these works [Pinçon et al., 1997; Schuck et al., 1998; Bonnell et al., 1998; Delory et al., 1998] all demonstrate that the local phase velocity of spectral components above and below the ambient LHR are anti-parallel. The discontinuity in the direction of the phase velocity at the ambient LHR is the hallmark of Hall physics in a density gradient; this delineates the boundary between trapped (in the electrostatic approximation) and propagating modes [Schuck et al., 1998; Schuck, 1999].

[7] The physical dimensions and wave properties of LHSS are summarized in Figure 1: a localized envelope of lower hybrid waves in a cigar-shaped field aligned density depletion. The relevant scale lengths are $\ell_{z}$ and $\ell_{\perp}$ respectively parallel and perpendicular to the geomagnetic field $B_{0}$. The semi-circular lines denote the azimuthal phase

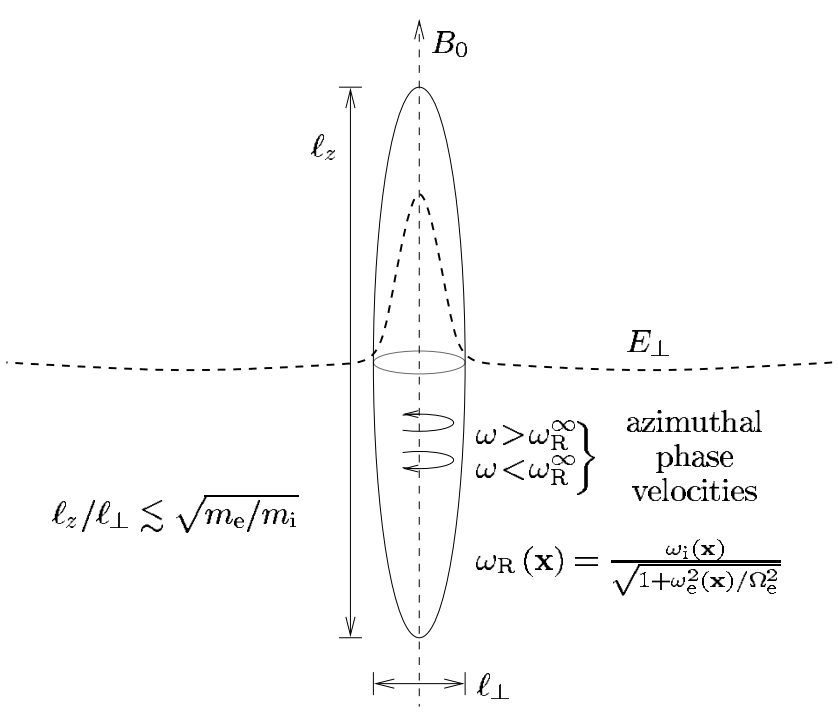

Figure 1. Schematic diagram of a LHSS: a localized envelope of lower hybrid waves in a cigar-shaped fieldaligned density depletion. The relevant scale lengths are $\ell_{z}$ and $\ell_{\perp}$ respectively parallel and perpendicular to the geomagnetic field $B_{0}$. The semi-circular lines denote the azimuthal phase velocity of right-handed (left-handed) fluctuations above (below) the ambient LHR $\omega_{\mathrm{R}}^{\infty}$.

velocity of right-handed (left-handed) fluctuations above (below) the ambient LHR $\omega_{\mathrm{LH}}(\infty)$.

[8] The TOPAZ III, AMICIST, PHAZE II and GEODESIC sounding rockets [Arnoldy et al., 1992; Kintner et al., 1992; Vago et al., 1992; Kintner, 1992; Lynch et al., 1994, 1996, 1999; Knudsen et al., 2001] established LHSS as a source of transversely accelerated ions contributing to the overall ion fluxes moving upward out of the auroral ionosphere [Shelley et al., 1976; Mizera and Fennell, 1977; Ghielmetti et al., 1978; Whalen et al., 1978; Klumpar, 1979; Shelley, 1979; Horwitz, 1980; Gorney et al., 1981]. In particular, Lynch et al. [1999] show that the energetic ion flux is consistent with trajectories which are only partially contained within the wave event. This suggests that the heating mechanism is local transit time acceleration by a coherent wave packet [Melatos and Robinson, 1993, 1996].

[9] There are several competing explanations for LHSS. Chang [1993] and Shapiro et al. [1993, 1995] propose that LHSS are a consequence of the modulational instability and collapse of lower hybrid waves. Although the MARIE and TOPAZ III observations initially supported this theory, subsequent statistical analysis discount many of its predictions [Pécseli et al., 1996, 1997; Kjus et al., 1997, 1998; Høymork et al., 2000, 2001]. These studies compare Freja observations of LHSS with the predictions of collapse theory and conclude that the collapse theory "in its simplest form" is incompatible with Freja data set [Pécseli et al., 1996, p. 5299]. This conclusion is based on the large discrepancies between the theoretical predictions and the observed structure widths, density depths, electric field strengths, and pertinent time-scales of LHSS. Robinson [1999] rationalizes these discrepancies using the modern theory of strong turbulence, a four phase nucleation cycle [Doolen et al., 1985; Robinson, 1996, 1999]: nucleation, 
collapse, arrest, and relaxation. However, a detailed analysis of the amplitude probability distribution of the electric field in regions containing LHSS discounts this more sophisticated collapse theory [Schuck et al., 2002]. Presently, the community is suggesting alternative explanations for LHSS because the discrepancies between collapse theory and observations are so numerous.

[10] Seyler [1994], Schuck et al. [1998], and Schuck [1999] suggest that the primary spectral features of LHSS electric fields are predicted by a linear theory of lower hybrid waves in a preexisting cylindrical density depletion. The essential feature of this linear description is the Hall current of the lower hybrid field produced by the electron $\mathbf{E} \times \mathbf{B}$ drift across the gradient of the density depletion. This current is not canceled by the ions which are unmagnetized near the lower hybrid frequency. The Hall current has three observable consequences: (1) The phase fronts of lower hybrid waves propagate azimuthally about the magnetic field at the center of LHSS. (2) Right-handed free modes propagate only above the ambient LHR within the cylindrical density depletion. Left-handed bound modes propagate only below the ambient LHR within the cylindrical density depletion. The direction of the phase velocity is discontinuous at the ambient LHR. (We note that if the Hall current is neglected, then right and left-handed modes are degenerate; both propagate above and below the ambient LHR within the cylindrical density depletion.) (3) The Hall current permits modes to propagate below the local LHR $\omega_{\mathrm{R}}(\mathbf{x})$ within the density depletion. The predictions of linear theory [Seyler, 1994; Schuck et al., 1998; Schuck, 1999] are in agreement with sounding rocket interferometric measurements and spectral analysis of LHSS electric fields [Pinçon et al., 1997; Schuck et al., 1998; Bonnell et al., 1998; Delory et al., 1998; Kintner et al., 2000].

[11] Ergun et al. [1995], Pécseli et al. [1996, 1997], Kjus et al. [1997, 1998], and Høymork et al. [2000, 2001] propose that the LHSS electric fields can be described by a WKBJ (Wenzel-Kramers-Brillouin-Jeffreys) [Jeffreys, 1923; Wentzel, 1926; Kramers, 1926; Brillouin, 1926] analysis of lower hybrid waves (electrostatic whistlers) propagating though a preexisting density depletion. This explanation "presumes that the density depletions are preexisting and the correlation between lower hybrid wave activity and the perturbations in density are solely caused by the change in refractive index of the waves" [Kjus et al., 1998, p. 26,647]. The WKBJ analysis proposed by Ergun et al. [1995], Bamber et al. [1995], Pécseli et al. [1996], and Høymork et al. [2000] is formulated on the homogeneous plasma dispersion relation (HPDR). This formulation presumes that density gradients do not change the essential propagation characteristic of lower hybrid waves. These WKBJ analyses reproduce the localization of lower hybrid waves in density depletions but, only under certain restrictive conditions. Ergun et al. [1995] show that a preferential depletion of hydrogen in a multi-species oxygen-dominated plasma lowers the local LHR causing lower hybrid waves to refract into the density depletion. This mechanism can produce bound states well below the ambient lower hybrid resonance, but is limited to low altitudes where hydrogen is the minority species. Bamber et al. [1995] conclude that wave refraction in a single species plasma has little influence on lower hybrid waves, stating "Ray tracing analysis shows that the electrostatic whistlers simply pass through density depletions, refracting slightly at the boundaries" (p. 23,809). Pécseli et al. [1996] and Høymork et al. [2000] disagree, suggesting that lower hybrid waves are refracted into density depletions. Their analysis is constrained to regions where the plasma density is low $\omega_{\mathrm{e}} \ll \Omega_{\mathrm{e}}$ and the LHR is sensitive to density variations. None of these wave refraction mechanisms explain the salient spectral features of LHSS wave measurements such as the discontinuity is the direction of the phase velocity at the ambient LHR and the anomalously low frequencies of bound states below the local LHR which have been observed in a broad range of altitudes under a variety of plasma conditions.

[12] Including the effects of the electron Hall current in a WKBJ analysis is possible and this has a profound influence on wave propagation. The manuscript is organized as follows: Section 2 discusses deficiencies of WKBJ analysis of LHSS formulated on the HPDR. Section 3 corrects the plasma dispersion relation by including the Hall current. Section 4 uses these results to perform a ray-tracing analysis of LHSS presenting calculations with and without the Hall term. This juxtaposition demonstrates that the Hall current dominates the usual refractive effects produced by the change in the local LHR $\omega_{R}(\mathbf{x})$. This ray-tracing analysis formulated with the Hall current agrees qualitatively with observations [Pinçon et al., 1997; Schuck et al., 1998; Bonnell et al., 1998; Delory et al., 1998; Kintner et al., 2000], and more formal eigenmode [Seyler, 1994; Schuck et al., 1998] and scattering analysis [Schuck, 1999] thus providing a unified description of the LHSS field structure.

\section{Previous Ray-Tracing Analyses of LHSS}

[13] Previous ray-tracing analyses of lower hybrid waves were formulated on the HPDR; either the Appleton-Hartree dispersion relation [Bamber et al., 1995] or the approximate plasma dispersion relation [Pécseli et al., 1996; Høymork et al., 2000]

$$
\omega(\mathbf{k}, \mathbf{x})=\omega_{\mathrm{R}}(\mathbf{x})\left(1+\frac{\mathcal{R} k_{z}^{2}}{2 k_{\perp}^{2}}+\frac{k_{\perp}^{2} R^{2}}{2}\right)
$$

where

$$
R \equiv \sqrt{\frac{3 T_{\mathrm{i}}}{\omega_{\mathrm{R}}^{2} m_{\mathrm{i}}}+\frac{2 T_{\mathrm{e}}}{\Omega_{\mathrm{e}}^{2} m_{\mathrm{e}}} \frac{\omega_{\mathrm{e}}^{2}}{\omega_{\mathrm{e}}^{2}+\Omega_{\mathrm{e}}^{2}}}
$$

is the dispersion scale length and the geomagnetic field is aligned with the $\hat{z}$-axis. In writing (2), $k_{z} / k_{\perp} \ll \sqrt{m_{\mathrm{e}} / m_{\mathrm{i}}}$ and $k_{\perp}^{2} R^{2} \ll 1$ has been implicitly assumed, the small electromagnetic correction has been neglected, $\mathcal{R}=m_{\mathrm{e}} / m_{\mathrm{i}}$, and $T_{j}$ is the temperature. (Note that lower hybrid waves do not satisfy Fermat's principle of least time because the dispersion relation is not a homogeneous function of $\mathbf{k}$ and $\omega$ [Weinberg, 1962].)

[14] The equations of the ray are obtained by the method of characteristics [Weinberg, 1962; Swansen, 1989]

$$
\begin{gathered}
\frac{d \mathbf{x}}{d t}=\nabla_{\mathbf{k}} \omega(\mathbf{k}, \mathbf{x}), \\
\frac{d \mathbf{k}}{d t}=-\nabla_{\mathbf{x}} \omega(\mathbf{k}, \mathbf{x}) .
\end{gathered}
$$


[15] These form a set of Hamiltonian equations where $\omega(\mathbf{k}, \mathbf{x})$, the time-independent "Hamiltonian," is a constant of the motion. In analogy with classical and quantum mechanics two states or types of rays are possible when the Hamiltonian is inhomogeneous. These types are distinguished by their frequency relative to the ambient LHR $\omega_{R}^{\infty} \equiv \omega_{R}(\infty)$ : (1) free rays/waves: propagate at frequencies above the ambient LHR $\omega>\omega_{R}^{\infty}$. These rays/waves can propagate in the ambient plasma but may be temporarily "localized" in density inhomogeneities. (2) bound rays/ waves: propagate at frequencies below the ambient LHR $\omega<\omega_{R}^{\infty}$. These rays/waves cannot propagate in the ambient plasma and are trapped in density inhomogeneities.

[16] We begin the WKBJ analysis of LHSS by reproducing and discussing the ray tracing results of Pécseli et al., [1996] using (1)-(4). Pécseli et al. [1996] computes twodimensional ray trajectories in the $x z$-plane. Høymork et al. [2000] has extended these calculations to three spatial dimensions. The inhomogeneity in the $y$-direction permits rays to be refracted out of the $x y$-plane. However, the essential refraction and localization mechanism of the HPDR is captured in two dimensions.

[17] Figure 2 shows a ray tracing analysis of free lower hybrid waves $\omega>\omega_{R}^{\infty}$ in a $0.5 \%$ Gaussian density depletion

$$
\widetilde{n}(\mathbf{x})=1+\Delta \exp \left(-\frac{x^{2}+y^{2}}{2 \ell_{\perp}}-\frac{z^{2}}{2 \ell_{z}^{2}}\right) .
$$

[18] Here $\tilde{n}(\mathbf{x})=n(\mathbf{x}) / n_{\infty}$ is normalized background plasma density profile where $n_{\infty}$ is the ambient plasma density, with $\Delta=-0.005$ and $1 / e$ width of $2 \mathrm{~km}$ $\left(\ell_{z}=\sqrt{2} / 2 \mathrm{~km}\right)$ along the magnetic field, and $150 \mathrm{~m}$ $\left(\ell_{\perp}=75 / \sqrt{2} \mathrm{~m}\right)$ in the perpendicular direction. The dashed lines indicate equidensity contours for the magnitude of the density depletion $\left(e^{-3}, e^{-2}, e^{-1}\right)$. The plasma parameters for the plots are $n_{\infty}=5 \times 10^{8} \mathrm{~m}^{-3}, B_{0}=40 \mu \mathrm{T}$, $R=0.34 \mathrm{~m}$, and $\mathcal{R}=12256$ representing an oxygen plasma with $9.3 \%$ hydrogen which corresponds to the composition of the topside $(1000 \mathrm{~km})$ auroral ionosphere. The initial values of the wave vectors and frequencies in the top panel of Figure 2 are $k_{x}=-0.5, k_{y}=0, k_{z}=4 \times 10^{-5}, 3.5 \times$ $10^{-5}, 3 \times 10^{-5} \mathrm{~m}^{-1}$, and $\omega / \omega_{\mathrm{R}}^{\infty}=1.013053,1.013044$, 1.013036 , respectively, at a position $(x, y, z)=(200,0,0) \mathrm{m}$. The wave vector in the bottom panel of Figure 2 is given by $k_{x}=-0.5, k_{y}=0$, and $k_{z}=3 \times 10^{-5} \mathrm{~m}^{-1}$ for initial positions $x=200, y=0$, and $z=300,-1300,-2900 \mathrm{~m}$ respectively.

[19] The tick marks on the ray trajectories indicate the magnitude (in arbitrary units) and direction of the local wave vector (phase velocity). The distance between tick marks is an indication of the magnitude of the local group velocity. For these rays, the $\hat{x}$-component of phase velocity and $\hat{x}$-component of group velocity (ray-trajectory) are always in the same direction. The "forward" propagation of these waves in the work of Pécseli et al. [1996] is caused by the thermal dispersion $k_{\perp}^{2} R^{2} \sim 0.03$ which dominates the electron inertial term $k_{z}^{2} / k_{\perp}^{2} \mathcal{R} \lesssim 8 \times 10^{-5}$. The lower hybrid rays for this example propagate across the magnetic field via thermal fluctuations. Laboratory experiments usually observe lower hybrid fluctuations as "backward-propagating" waves in the perpendicular plane; this component of the group velocity is in the opposite direction as the

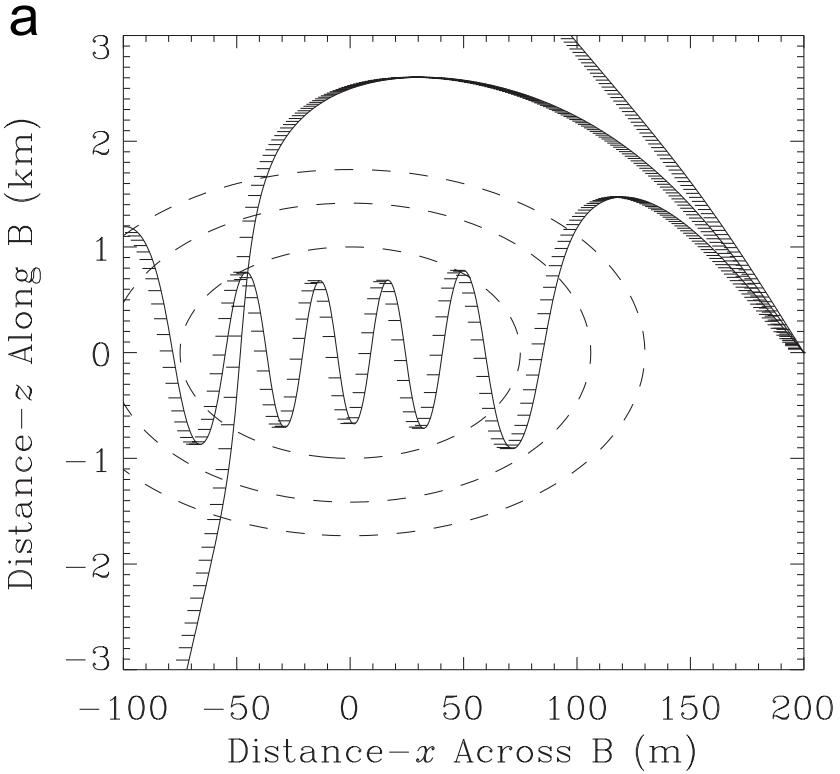

b

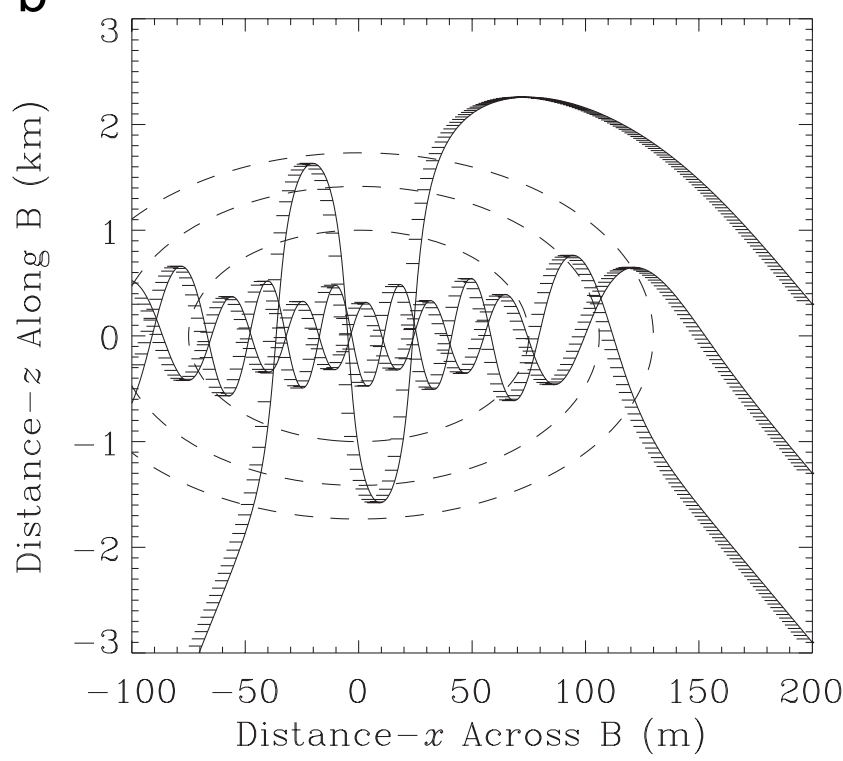

Figure 2. (a, b) A ray tracing analysis of free lower hybrid waves $\omega>\omega_{R}^{\infty}$ in a $0.5 \%$ Gaussian density depletion $(\Delta=$ -0.005 ) with a $1 / e$ width of $2 \mathrm{~km}$ along the magnetic field and $150 \mathrm{~m}$ in the across the magnetic field. The ray trajectories are determined from the HPDR and (1)-(5). The dashed lines indicate equidensity contours for the magnitude of the density depletion $\left(e^{-3}, e^{-2}, e^{-1}\right)$. See text for plasma parameters and initial conditions for the rays. After Pécseli et al. [1996].

phase velocity. [Stenzel and Gekelman, 1975; Bellan and Porkolab, 1975; Bamber et al., 1994, 1995; Rosenberg and Gekelman, 1998, 2000, 2001]. Space observations are consistent with dominance of inertial dispersion over thermal dispersion (see point 2 below). Furthermore Santolik and Gurnett [2002] recently demonstrated that auroral hiss at high altitudes propagates "backward" perpendicular to the magnetic field in accordance with cold plasma theory. 
[20] Focusing and localization of the rays are apparent; rays are reflected multiple times from the ends of the cavity (along the magnetic field) as they cross the cavity (perpendicular to the magnetic field). This suggests that localization is caused by wave reflection in the $\hat{z}$-direction since the group velocity of the lower hybrid waves actually increases in the density depletion. We note that reflection in the $\hat{z}$-direction has been biased by the choice of wave numbers. In the perpendicular direction the usual WKBJ criterion $k_{\perp}$ $\ell_{\perp} \approx 26.5 \gg 1$ is well satisfied but along the magnetic field $k_{z} \ell_{z} \approx 0.028 \ngtr 1$. This disparity between the perpendicular and parallel criteria $k_{\perp} \ell_{\perp} /\left(k_{z} \ell_{z}\right) \gtrsim 120$ biases the results in favor of focusing by parallel effects, i.e., reflection along the magnetic field. Høymork et al. [2000] considers different initial wave vectors $k_{\perp} \ell_{\perp} \approx 5.3 \gg 1$ and $k_{z} \ell_{z} \approx 0.0014 \gg 1$ but the disparity is similar $k_{\perp} \ell_{\perp} /\left(k_{z} \ell_{z}\right) \sim 4000$. Increasing the parallel scale of the density depletion decreases the focusing effects shown in Figure 2.

[21] The frequencies associated with the rays shown in Figure 2 are only $1.3 \%$ above the ambient LHR $\omega \approx 1.013$ $\omega_{\mathrm{R}}^{\infty}$ and extremely oblique $k_{z} / k_{\perp}=0.00008 \ll \sqrt{m_{\mathrm{e}} / m_{\mathrm{i}}}$ $\sim 0.009$ even for lower hybrid waves. These results are not characteristic of localized waves associated with LHSS at sounding rocket altitudes which often exhibit significant spectral content well above the ambient LHR and bound states at approximately $1 / 2$ the ambient LHR with relatively long perpendicular wavelengths $\lambda \sim 30-50 \mathrm{~m}$ [Vago et al., 1992; Pinçon et al., 1997; Schuck et al., 1998; Bonnell et al., 1998]. Spectral analysis of Freja LHSS exhibit similar features well away from the inferred local LHR [Pécseli et al., 1996, 1997, Kjus et al., 1997, 1998; Høymork et al., 2000, 2001]. Equation (2) must be corrected for substantial values of $k_{z}\left(k_{z}^{2} / k_{\perp}^{2} \mathcal{R} \sim 1\right)$ and the Hall current in order to account for the full frequency regime observed.

[22] Høymork et al. [2000] assert that this ray-tracing analysis "demonstrated the possibility of trapped lower hybrid modes in a localized density depletion." Trapped modes are bound states in the electrostatic approximation which propagate within the density depletion below the ambient LHR. While the existence of bound states may be predicted using WKBJ analysis formulated on the HPDR, the spectral extent of these states is extremely limited. Under (2), bound states may only propagate above the minimum LHR frequency (at $\mathbf{x}=0$ for (5)) and below ambient LHR: $\omega_{R}^{\infty} \geq \omega(\mathbf{k}, \mathbf{x}) \geq \omega_{R}(0)$. Therefore, the minimum frequency of a bound state is

$$
\omega_{\mathrm{R}}^{\infty} \geq \omega(\mathbf{k}, \mathbf{x}) \geq \omega_{\mathrm{R}}^{\infty}\left[1+\frac{\Omega_{\mathrm{e}}^{2} \Delta / 2}{\omega_{\mathrm{e}}^{2}(\infty)+\Omega_{\mathrm{e}}^{2}}\right] .
$$

[23] Consequently, for a density depletion of $2 \%(\Delta=$ -0.02 ) this predicts that the minimum frequency of a trapped mode is only $1 \%$ below the ambient LHR which is inconsistent with sounding rocket and Freja observations. Consider Figure 7 of Kjus et al. [1998] (see also Figure 3 of Pécseli et al. [1997]). This figure shows an example of an LHSS observed by the Freja satellite containing a bound state centered at $2500 \mathrm{kHz}$. The ambient LHR determined from the spectral cutoff frequency at the edge of the data set is approximately $3 \mathrm{kHz}$ and the density depletion is approximately $2.5 \%$. The minimum frequency of a bound state under the WKBJ analysis based on (2) is $2960 \mathrm{kHz}$. To account for this bound state using the HPDR the density depletion depth would necessarily be $30 \%$ not $2.5 \%$. A $30 \%$ density depletion would be extreme according to Freja observations. Indeed, no such depths are reported in the Freja literature [Dovner et al., 1994; Eriksson et al., 1994; Pécseli et al., 1996, 1997; Dovner et al., 1997; Kjus et al., 1997, 1998; Knudsen et al., 1998; Høymork et al., 2000, 2001]. A similar discrepancy between the predictions of WKBJ theory formulated on the HPDR and Freja observations of trapped modes is apparent in Figure 1 of Pécseli et al. [1996] and Figure 1 of Høymork et al., [2000] (see also Figure 1 in Høymork et al. [2001]). Thus, we conclude that WKBJ analysis [Pécseli et al., 1996; Høymork et al., 2000] of bound states using the HPDR is inconsistent with Freja observations of bound states.

[24] The WKBJ ray-tracing analysis formulated on the HPDR suffers from several important deficiencies when applied to LHSS:

[25] 1. This analysis is misleading because it suggests that the primary mechanism of localization for LHSS waves is refractive focusing along the magnetic field. However, the Hall current in the perpendicular plane dramatically affects the dispersive characteristics of lower hybrid waves in an inhomogeneous plasma [Seyler, 1994; Schuck et al., 1998; Schuck, 1999]. Indeed most of the spectral properties of LHSS waves are quantitatively predicted while including Hall effect but neglecting both variations in the local LHR $\omega_{\mathrm{R}}(\mathbf{x})$ and thermal effects [Pinçon et al., 1997; Schuck et al., 1998; Bonnell et al., 1998; Delory et al., 1998; Kintner et al., 2000; Schuck, 1999].

[26] 2. Pécseli et al. [1996] and Høymork et al., [2000] investigate the nearly two-dimensional regime where the thermal dispersion dominates the electron inertial term $k_{z}^{2} / k_{\perp}^{2} \mathcal{R} \ll k_{\perp}^{2} R^{2} \ll 1$. However, this regime does not represent observations where localized states were observed well above the ambient LHR. If free localized states with $\omega \approx \sqrt{2} \omega_{\mathrm{R}}$ are coupled to propagating modes with $k_{\perp}^{2} R^{2} \ll 1$ then these propagating modes must have $k_{z}^{2} / k_{\perp}^{2} \mathcal{R} \sim 1$ in the ambient plasma; this suggests that the appropriate regime is $k_{\perp}^{2} R^{2} \ll k_{z}^{2} / k_{\perp}^{2} \mathcal{R} \sim 1$.

[27] 3. This analysis cannot reasonably account for frequencies of "bound" or "trapped" states below the local LHR of the density depletion [Pinçon et al., 1997; Schuck et al., 1998; Bonnell et al., 1998; Delory et al., 1998; Kintner et al., 2000; Pécseli et al., 1996, 1997; Kjus et al., 1997, 1998; Høymork et al., 2000, 2001].

[28] 4. WKBJ analysis is expected to break down when the gradient scale length $L$ is small compared to a wavelength: $k L \lesssim 1$. Experiments show that the longest wavelengths of lower hybrid waves localized in density depletions are on the order of the cavity diameter $\lambda \sim$ $2 \ell_{\perp} \sim$ 40-50 m. [Pinçon et al., 1997; Schuck et al., 1998; Bonnell et al., 1998; Kintner et al., 2000]. Furthermore, the wavelengths of ambient fluctuations are much longer than the cavity diameter $\lambda \gg \ell_{\perp}$ [Vago et al., 1992].

[29] The mathematical breakdown of WKBJ cannot be rectified for $k L \lesssim 1$. However, in practice ray-tracing often provides correct zeroth order results even in regimes where geometrical optics is expected to fail. This remarkable property is utilized to investigate lower hybrid current drive in fusion devices [Bizarro, 1993; Bizarro and Moreau, 1993; Bizarro et al., 1993] and describe the propagation of 
electron-Bernstein waves [Armstrong et al., 1984]. In particular, ray-tracing correctly predicts the behavior and direction of the local group velocity, the direction of the local wave vector and general regions of enhanced wave amplitude in the vicinity of caustics [Armstrong et al., 1984]. Consequently, correcting the previous LHSS ray-tracing analyses to account for the Hall effects should provide consistency between WKBJ analysis, observations [Pinçon et al., 1997; Schuck et al., 1998; Bonnell et al., 1998; Delory et al.,1998; Kintner et al., 2000], and more formal eigenmode [Seyler, 1994; Schuck et al., 1998] and scattering analysis [Schuck, 1999] providing a unified explanation for the LHSS field structure. We demonstrate that important properties of the LHSS electric field may be qualitatively described by a raytracing analysis when the dispersion relation is formulated upon the Hall current physics.

\section{Model for Electrostatic Lower Hybrid Waves}

[30] Below we describe a linear model for electrostatic lower hybrid propagation in the presence of an ambient plasma density inhomogeneity. The equations for the ions are

$$
\begin{gathered}
\partial_{t} n_{\mathrm{i}}+\nabla_{\perp} \cdot\left(n \mathbf{u}_{\mathrm{i} \perp}\right)=0, \\
m_{\mathrm{i}} \partial_{t} \mathbf{u}_{\mathrm{i} \perp}=-e \nabla_{\perp} \varphi-\frac{\gamma_{\mathrm{i}} T_{\mathrm{i}}}{n} \nabla_{\perp} n_{\mathrm{i}},
\end{gathered}
$$

and the equations for the electrons are

$$
\begin{gathered}
\partial_{t} n_{\mathrm{e}}+\nabla \cdot\left(n \mathbf{u}_{\mathrm{e}}\right)=0 \\
m_{\mathrm{e}} \partial_{t} \mathbf{u}_{\mathrm{e}}=e \nabla \varphi-\frac{e}{c}\left(\mathbf{u}_{\mathrm{e}} \times \mathbf{B}_{0}\right)-\frac{\gamma_{\mathrm{e}} T_{\mathrm{e}}}{n} \nabla_{\perp} n_{\mathrm{e}}
\end{gathered}
$$

[31] In writing (7) and (8) we neglect ion dynamics and thermal effects along the magnetic field. The system is closed with Poisson's equation

$$
\nabla_{\perp}^{2} \varphi=-4 \pi e\left(n_{\mathrm{i}}-n_{\mathrm{e}}\right) .
$$

[32] Assuming harmonic time dependence, the ion velocity and density fluctuations are

$$
\mathbf{u}_{\mathrm{i} \perp}=-\frac{i}{\omega}\left(\frac{e}{m_{\mathrm{i}}} \nabla_{\perp} \varphi+\frac{\gamma_{\mathrm{i}} T_{\mathrm{i}}}{m_{\mathrm{i}} n} \nabla_{\perp} n_{\mathrm{i}}\right),
$$

and

$$
\left(\omega^{2}+\frac{\gamma_{\mathrm{i}} T_{\mathrm{i}}}{m_{\mathrm{i}}} \nabla_{\perp}^{2}\right) n_{\mathrm{i}}=-\frac{e}{m_{\mathrm{i}}} \nabla_{\perp} \cdot\left(n \nabla_{\perp} \varphi\right) .
$$

[33] The electron velocity fluctuations are

$$
\begin{aligned}
\mathbf{u}_{\mathrm{e}} & =\frac{\omega^{2} \mathbf{W}_{\perp}}{\omega^{2}-\Omega_{\mathrm{e}}^{2}}-\frac{i \Omega_{\mathrm{e}} \omega \mathbf{W}_{\perp} \times \hat{z}}{\omega^{2}-\Omega_{\mathrm{e}}^{2}}+\frac{i}{\omega} \frac{e}{m_{\mathrm{e}}} \partial_{z} \varphi \hat{z} . \\
& \approx-\frac{\omega^{2}}{\Omega_{\mathrm{e}}^{2}} \mathbf{W}_{\perp}+i \frac{\omega}{\Omega_{\mathrm{e}}} \mathbf{W}_{\perp} \times \hat{z}+\frac{i}{\omega} \frac{e}{m_{\mathrm{e}}} \partial_{z} \varphi \hat{z},
\end{aligned}
$$

where

$$
\mathbf{W}_{\perp}=\frac{i}{\omega}\left(\frac{e}{m_{\mathrm{e}}} \nabla_{\perp} \varphi-\frac{\gamma_{\mathrm{e}} T_{\mathrm{e}}}{m_{\mathrm{e}} n} \nabla_{\perp} n_{\mathrm{e}}\right)
$$

and $\omega \ll \Omega_{\mathrm{e}}$ has been assumed. Above $n=n(\mathbf{x})$ is a timeindependent quasineutral density inhomogeneity. Inserting (11a) into (8a) we obtain

$$
\begin{aligned}
\omega^{2}\left(1-\frac{1}{\Omega^{2}} \frac{\gamma_{\mathrm{e}} T_{\mathrm{e}}}{m_{\mathrm{i}}} \nabla_{\perp}^{2}\right) n_{\mathrm{e}}= & -\frac{\omega^{2}}{\Omega^{2}} \frac{e}{m_{\mathrm{i}}} \nabla_{\perp} \cdot\left(n \nabla_{\perp} \varphi\right)+i \frac{\omega}{\Omega_{\mathrm{e}}} \frac{e}{m_{\mathrm{e}}} \nabla_{\perp} \\
& \cdot\left(n \nabla_{\perp} \varphi \times \hat{z}\right)+\frac{e}{m_{\mathrm{e}}} \partial_{z}\left(n \partial_{z} \varphi\right) .
\end{aligned}
$$

[34] Inserting (10b) and (11) into (9), letting $\varphi=\varphi(x)$ $e^{i \psi(\mathbf{x})}$ and $\nabla \psi(\mathbf{x}) \equiv i \mathbf{k}(\mathbf{x})$, and retaining only the lowest order WKBJ approximation (see section 6.4 of Swansen [1989] for the details of the WKBJ method.) we obtain the local dispersion relation for lower hybrid waves propagating in a plasma density inhomogeneity

$$
\omega(\mathbf{k}, \mathbf{x})=\omega_{\mathrm{LH}}(\mathbf{k}, \mathbf{x})\left[1+\frac{1}{2} k_{\perp}^{2} \mathcal{C}^{2}(\mathbf{k}, \mathbf{x})\right]
$$

where $k_{\perp}^{2} \mathcal{C}^{2}(\mathbf{k}, \mathbf{x}) \ll 1$,

$$
\omega_{\mathrm{LH}}(\mathbf{k}, \mathbf{x})=\mathcal{H} \pm \sqrt{\mathcal{H}^{2}+\omega_{\mathrm{R}}^{2}\left(1+\mathcal{R} k_{z}^{2} / k_{\perp}^{2}\right)}
$$

is the cold plasma lower hybrid frequency,

$$
\mathcal{H} \equiv \mathcal{H}\left(\mathbf{k}_{\perp}, \mathbf{x}\right)=\frac{\mathcal{R} \omega_{\mathrm{R}}^{2}}{2 \Omega_{\mathrm{e}} k_{\perp}^{2}}\left(k_{y} \partial_{x}-k_{x} \partial_{y}\right) \log \widetilde{n}(\mathbf{x})
$$

is the Hall frequency shift caused by the electron $\mathbf{E} \times \mathbf{B}$ drift over a density gradient, $\omega_{\mathrm{R}} \equiv \omega_{\mathrm{R}}(\mathbf{x})$, and

$$
\mathcal{C}^{2}(\mathbf{k}, \mathbf{x}) \approx 2 \frac{\frac{\gamma_{\mathrm{i}} T_{\mathrm{i}} \omega_{\mathrm{R}}^{2}}{m_{\mathrm{i}} \omega_{\mathrm{LH}}^{2}}+\frac{\gamma_{\mathrm{e}} T_{\mathrm{e}}}{m_{\mathrm{e}} \Omega_{\mathrm{e}}^{4}}\left[\Omega_{\mathrm{e}}^{2} \omega_{\mathrm{R}}^{2}+\omega_{\mathrm{LH}}^{2}\left(\omega_{\mathrm{R}}^{2} \mathcal{R}-\Omega_{\mathrm{e}}^{2}\right)\right]}{\omega_{\mathrm{LH}}^{2}+\omega_{\mathrm{R}}^{2}\left(1+\mathcal{R} k_{z}^{2} / k_{\perp}^{2}\right)}
$$

where $\mathcal{C}(\mathbf{k}, \mathbf{x})$ is the thermal dispersion scale length. Observe that the Hall term produces an explicit dependence on the relative direction between the perpendicular wave number $\mathbf{k}_{\perp}$ and the density gradient $\nabla \tilde{n}$, not simply the magnitude of the wave number $k_{\perp}$. Neglecting the Hall term and assuming $k_{z} / k_{\perp} \ll \sqrt{m_{\mathrm{e}} / m_{\mathrm{i}}}$ the dispersion scale length is

$$
\mathcal{C}(\mathbf{k}, \mathbf{x}) \approx \sqrt{\frac{\gamma_{\mathrm{i}} T_{\mathrm{i}}}{m_{\mathrm{i}} \omega_{\mathrm{R}}^{2}}+\frac{\gamma_{\mathrm{e}} T_{\mathrm{e}} \omega_{\mathrm{e}}^{2}}{m_{\mathrm{e}} \Omega_{\mathrm{e}}^{2}\left(\omega_{\mathrm{e}}^{2}+\Omega_{\mathrm{e}}^{2}\right)}}
$$

which is the result obtained by Shapiro et al. [1993, 1995] with $\gamma_{\mathrm{i}}=3$ and $\gamma_{\mathrm{e}}=2$, and used by Pécseli et al. [1996] and Høymork et al. [2000].

\section{Ray-Tracing Analysis of LHSS}

[35] The influence of the Hall current on lower hybrid waves propagating in a cylindrical density depletion may be investigated using equations (1), (4)-(5), and (12). We consider plasma parameters appropriate to Freja altitudes estimated from the average values reported in Table 1 of Høymork et al. [2000] and average values reported for orbit 1234 of Kjus et al. [1998] and Høymork et al. [2000]. 

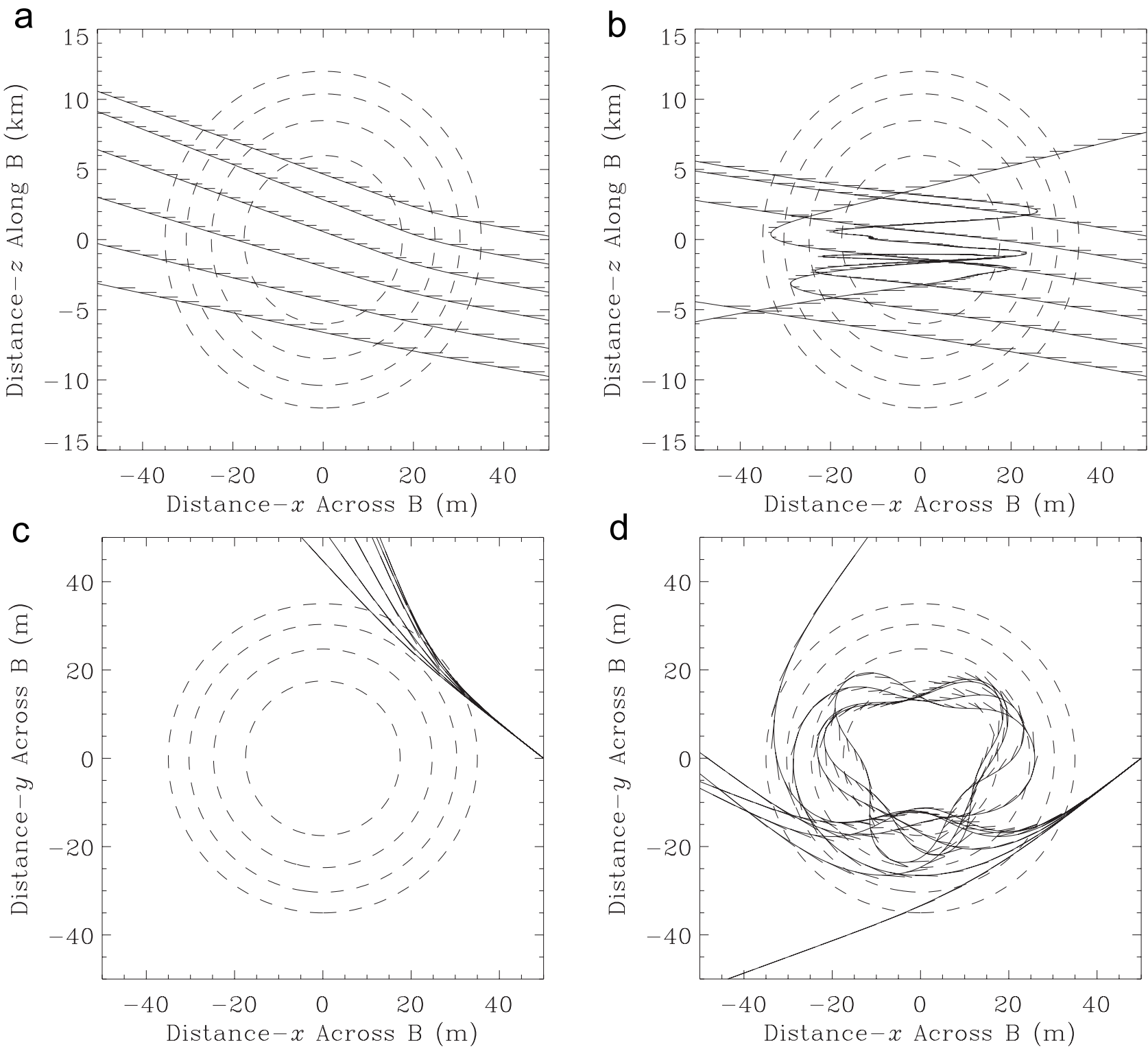

Figure 3. (a-d) A ray tracing analysis of free lower hybrid waves $\omega>\omega_{\mathrm{R}}^{\infty}$ in a $2 \%$ Gaussian density depletion $(\Delta=-0.02)$ with a $1 / e$ width of $12 \mathrm{~km}$ along the magnetic field and $35 \mathrm{~m}$ in the perpendicular direction. The dashed lines indicate equidensity contours for the magnitude of the density depletion $\left(e^{-4}, e^{-3}, e^{-2}, e^{-1}\right)$. See text for the plasma parameters and initial conditions for the rays. (top) Projection of the rays onto the $x z$-plane. (bottom) Projection of the rays onto $x y$-plane. (left) Waves with a "lefthanded" phase velocity are reflected from of the cavity. (right) Waves with a "right-handed" phase velocity are refracted into the cavity; the phase fronts in the cavity appear to rotate in a right-handed sense about the geomagnetic field.

[36] Figure 3 shows a ray tracing analysis of free lower hybrid waves $\omega>\omega_{R}^{\infty}$ in a $2 \%$ Gaussian density depletion $(\Delta=-0.02)$ with a $1 / e$ width of $12 \mathrm{~km}$ along the magnetic field and $35 \mathrm{~m}$ in the perpendicular direction. The perpendicular scale size is consistent with mean value observed on Freja orbit 1234 (see Figure 6 of Høymork et al. [2000]). The dashed lines indicate equidensity contours for the magnitude of the density depletion $\left(e^{-4}, e^{-3}, e^{-2}, e^{-1}\right)$. The plasma parameters for the plots are: $n_{\infty}=500 \mathrm{~cm}^{-3}$, $B_{0}=30 \mu \mathrm{T}, T_{\mathrm{i}}=0.2 \mathrm{eV}, T_{\mathrm{e}}=0.25 \mathrm{eV}, \gamma_{\mathrm{i}}=3, \gamma_{\mathrm{e}}=2$, $\mathcal{C}(0, \infty)=0.5 \mathrm{~m}$, and $\mathcal{R}=6427,\left(f_{\mathrm{R}}=\omega_{\mathrm{R}}^{\infty} /(2 \pi)=2436\right.$ $\mathrm{Hz}$ ). (Here $\mathcal{R}$ was determined by estimating $f_{\mathrm{R}}$ from the electrostatic cutoff [Brice and Smith, 1964, 1965] of Plate 2a of Kjus et al. [1998] for orbit 1234.) The initial value of the wave vector is $k_{x}=4 \times 10^{-3}, k_{y}= \pm 3 \times 10^{-3}$, and $k_{z}=$ $1 \times 10^{-4} \mathrm{~m}^{-1}$ with $\omega / \omega_{\mathrm{R}}^{\infty}=2.0$ for initial positions $x=50$, $y=0, z=250,-1750,-3750,-5750,-9750,-7750 \mathrm{~m}$ respectively. For these wave vectors $k_{\perp} c / \omega_{\mathrm{e}} \sim 1.2, k_{\perp} \ell_{\perp}=$ $0.06, k_{z} \ell_{z}=0.46$, and $k_{\perp} \ell_{\perp} /\left(k_{z} \ell_{z}\right) \sim 0.14$. Since the WKBJ criterion $k L \gtrsim 1$ is not strictly satisfied parallel or perpendicular to the magnetic field, we emphasize that only qualitative results can be expected from this analysis.

[37] The top panels of Figure 3 show the projection of the rays onto the $x z$-plane and the bottom panels show the 
projection of the rays onto the $x y$-plane. The left panels correspond to the initial condition $k_{y}=-3 \times 10^{-3} \mathrm{~m}^{-1}$; these rays exhibit a "left-handed" phase velocity relative to the magnetic field at the center of the cavity and are reflected from the density depletion. The right panels correspond to the initial condition $k_{y}=3 \times 10^{-3} \mathrm{~m}^{-1}$; these rays exhibit a "right-handed" phase velocity relative to the magnetic field at the center of the cavity and are refracted into density depletion. The presence of the density cavity can have a dramatic effect on an incident unidirectional wave field producing a substantial scatter in the $x y$-plane. A large number of cavities may randomize the direction of the incident rays in agreement with the observations and ray-tracing calculations of Høymork et al. [2000]. However, whereas Høymork et al. [2000] conclude that the HPDR produced significant scatter in the forward direction, the Hall term can completely reverse the direction of an incident ray in the $x y$-plane producing considerable backscatter.

[38] For comparison, Figure 4 shows free lower hybrid waves $\omega>\omega_{\mathrm{R}}^{\infty}$ in a $2 \%$ Gaussian density depletion $(\Delta=$ -0.02 ). These results correspond to the same plasma configuration and initial conditions as Figures $3 \mathrm{a}$ and $3 \mathrm{~b}$ except the Hall term is neglected. When the Hall term is neglected, these rays "simply pass through density depletions, refracting slightly at the boundaries" [Bamber et al., 1995, p. 23,809]. The nearly straight ray trajectories may seem surprising. However these rays are nearly tangent to the $e^{-3}$ density contour where the density depletion is only $0.1 \%$ (20 times weaker than at the center of the cavity). The distinct differences between Figure 4 and Figures $3 \mathrm{a}$ and $3 \mathrm{~b}$ can only be attributed to the profound effect of the Hall current on the ray trajectories.

[39] Figure 5 shows a ray tracing analysis of bound lower hybrid waves $\omega<\omega_{\mathrm{R}}^{\infty}$ in a $2 \%$ Gaussian density depletion $(\Delta=-0.02)$ for the same plasma configuration as Figure 3. The initial value of the wave vector is $k_{x}=4 \times 10^{-3}, k_{y}=$ $4 \times 10^{-3}$, and $k_{z}=7.5 \times 10^{-5} \mathrm{~m}^{-1}, \omega / \omega_{\mathrm{R}}^{\infty}=0.71$ for the initial position $x=0, y=12.3, z=0 \mathrm{~m}$. The phase velocity is always in the "left-handed" direction about the magnetic field at the center of the density depletion. Under ray-tracing analysis formulated on the HPDR (where the Hall term is neglected) the minimum frequency for bound ray is $\omega_{R}(0) /$ $\omega_{\mathrm{R}}^{\infty}=0.99$ whereas under this analysis (including the Hall term) the minimum frequency for a bound ray is well below the local LHR. Thus, the Hall current allows lower hybrid waves to propagate in the density depletion at frequencies below the minimum local LHR of the plasma located at the center of the Gaussian density depletion.

\section{Discussion and Conclusions}

[40] According to equation (12) the waves in the density depletion should be observed with the following phase velocity properties: (1) for frequencies above the ambient LHR $\omega>\omega_{\mathrm{R}}^{\infty}$ the phase velocities are only right-handed; (2) for frequencies below the ambient LHR $\omega<\omega_{R}^{\infty}$ the phase velocities are only left-handed. These ray-tracing predictions are in complete agreement with eigenmode [Seyler, 1994; Schuck et al., 1998] and scattering theory [Schuck, 1999], and sounding rocket phase velocity measurements [Pinçon et al., 1997; Schuck et al., 1998; Bonnell et al.,
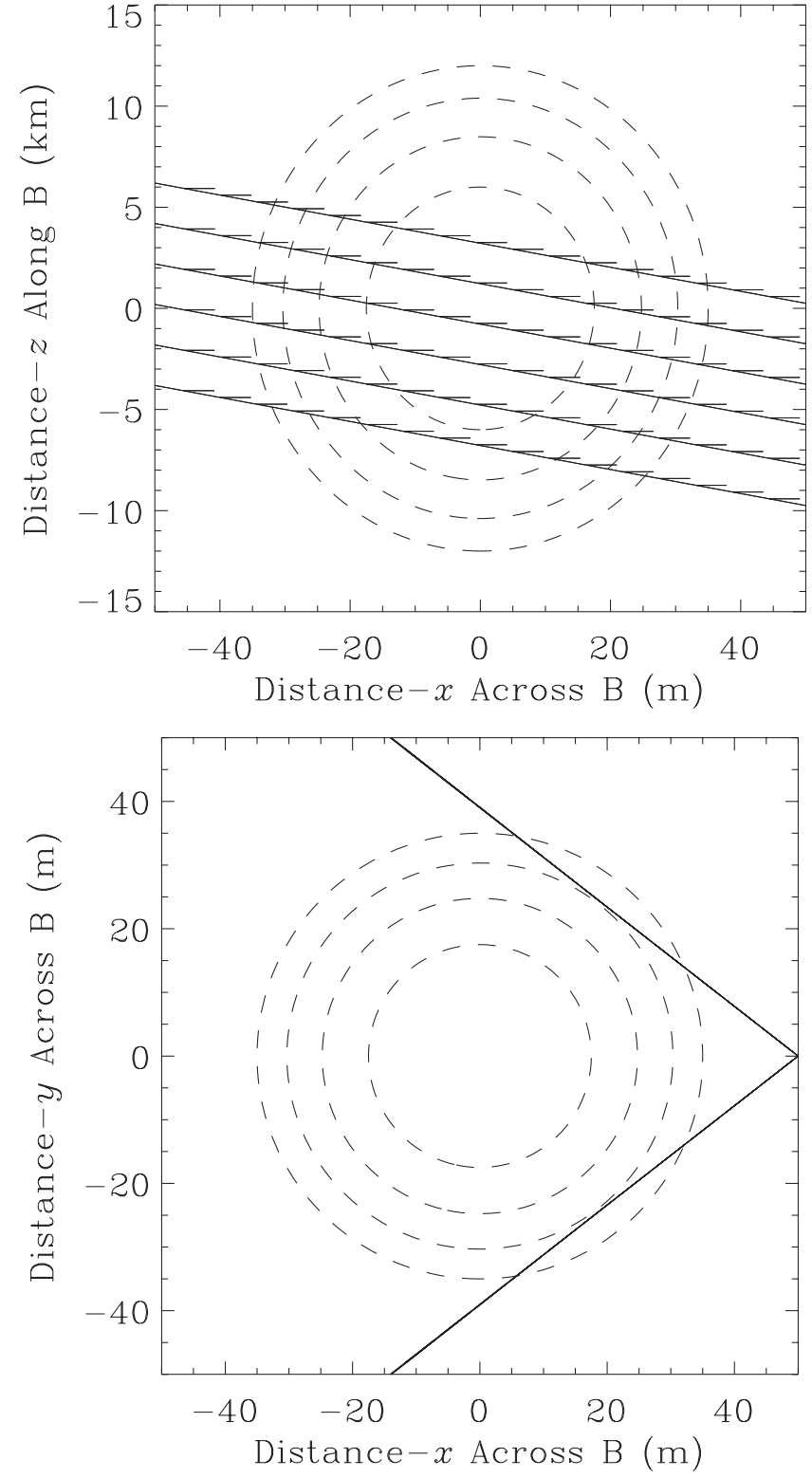

Figure 4. A ray tracing analysis of free lower hybrid waves $\omega>\omega_{R}^{\infty}$ in a $2 \%$ Gaussian density depletion $(\Delta=$ -0.02). Same plasma configuration, initial conditions, and data presentation as Figures $3 \mathrm{a}$ and $3 \mathrm{~b}$ except the Hall term is neglected. Note that the rays follow straight trajectories through the density depletion.

1998; Kintner et al., 2000]. This agreement seems to justify the extension of the ray-tracing analysis to the $k L<1$ regime for this situation. Furthermore the Hall term in (12) resolves the discrepancy between the observed frequencies of bound states [Pinçon et al., 1997; Schuck et al., 1998; Bonnell et al., 1998; Delory et al., 1998; Kintner et al., 2000; Pécseli et al., 1996, 1997; Kjus et al., 1998; Høymork et al., 2000, 2001] and the predictions of ray-tracing analysis based on the HPDR [Pécseli et al., 1996; Høymork et al., 2000]. While ray-tracing analysis cannot quantitatively predict bound state and resonant frequencies or wave amplitudes accessible using full-wave [Seyler, 1994; Schuck et al., 1998] and scattering theory [Schuck et al., 1998], ray- 

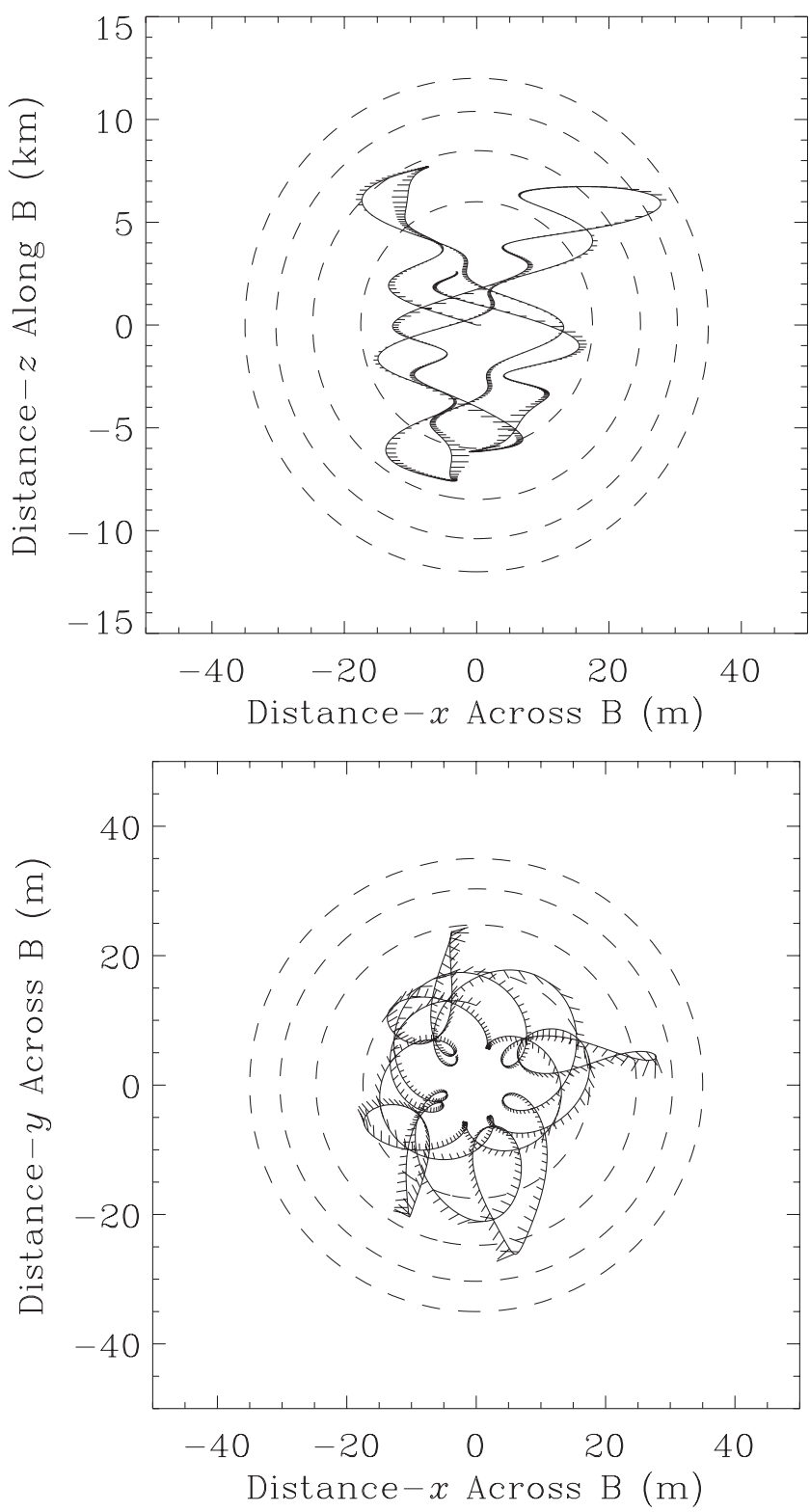

Figure 5. A ray tracing analysis of bound lower hybrid waves $\omega<\omega_{R}^{\infty}$ in a $2 \%$ Gaussian density depletion $(\Delta=$ -0.02 ). Same plasma configuration and data presentation as Figure 3 . The initial value of the wave vector is $k_{x}=4 \times$ $10^{-3}, k_{y}=4 \times 10^{-3}$, and $k_{z}=7.5 \times 10^{-5} \mathrm{~m}^{-1}$ for the initial position $x=0, y=12.3, z=0 \mathrm{~m}$. The phase velocity is always in the "left-handed" direction about magnetic field at the center of the density depletion.

tracing analysis formulated on the Hall current does produce qualitatively correct behavior for rays propagating above and below the ambient LHR consistent with observation and more sophisticated theories. These rays are profoundly different from those predicted by the HPDR. We conclude that the Hall current is an essential element of the theoretical explanation for the LHSS field structure.

[41] The Hall current is intrinsically connected with the scale of the density gradient. Indeed, the Hall term is independent of the absolute density depletion depth. This term may also be responsible lower hybrid waves localized in the gradients of density enhancements observed by McAdams et al. [1998] and Høymork et al. [2000]. We speculate that the Hall current may also play a role in other related situations such as lower hybrid current drive in fusion devices [Bizarro, 1993; Bizarro and Moreau, 1993; Bizarro et al., 1993] and the interaction of lightning induced or ducted whistlers at the boundaries of density irregulaties [Baker et al., 2000; Carpenter et al., 2002].

[42] Acknowledgments. Arthur Richmond thanks David Knudsen and another referee for their assistance in evaluating this paper.

\section{References}

Armstrong, R. J., Å. Fredriksen, H. L. Pécseli, and J. Trulsen, ElectronBernstein waves in inhomogeneous magnetic fields, Plasma Phys. Control. Fusion, 26(5), 703-716, 1984.

Arnoldy, R., K. Lynch, P. M. Kintner, J. Vago, S. Chesney, T. E. Moore, and C. J. Pollock, Bursts of transverse ion acceleration at rocket altitudes, Geophys. Res. Lett., 19(4), 413-416, 1992.

Baker, S. D., M. C. Kelley, C. M. Swenson, J. Bonnell, and D. V. Hahn, Generation of electrostatic emissions by lighting-induced whistler-mode radiation above thunderstorms, J. Atmos. Sol. Terr. Phys., 62(15), $1393-$ 1404, 2000.

Bamber, J. F., W. Gekelman, and J. E. Maggs, Whistler wave mode conversion to lower hybrid waves at a density striation: A laboratory investigation of an auroral process, Phys. Rev. Lett., 73(22), 2990-2993, 1994.

Bamber, J. F., J. E. Maggs, and W. Gekelman, Whistler wave interaction with a density striation: A laboratory investigation of an auroral process, J. Geophys. Res., 100, 23,795-23,810, 1995.

Bellan, P., and M. Porkolab, Excitation of lower hybrid waves by a slow wave structure, Phys. Rev. Lett., 34(3), 124-127, 1975.

Bizarro, J. P., On the dynamics of the launched power spectrum during lower hybrid current drive in tokamaks, Nucl. Fusion, 33(5), 831-834, 1993.

Bizarro, J. P., and D. Moreau, On ray stochasticity during lower-hybrid current drive in tokamaks, Phys. Fluids B, 5(4), 1227-1238, 1993.

Bizarro, J. P., et al., On self-consistent ray-tracing and Fokker-Plank modelling of the hard x-ray emission during lower-hybrid current drive in tokamaks, Phys. Fluids B, 5(9), 3276-3282, 1993.

Bonnell, J. W., P. W. Schuck, J.-L. Pinçon, C. E. Seyler, and P. M. Kintner, Observation of bound states and counter-rotating eigenmodes in the auroral ionosphere, Phys. Rev. Lett., 80(26), 5734-5737, 1998.

Brice, N. M., and R. L. Smith, A very-low-frequency plasma resonance, Nature, 203, 926, 1964.

Brice, N. M., and R. L. Smith, Lower hybrid resonance emissions, J. Geophys. Res., 70, 71-80, 1965.

Brillouin, L., La mécanique ondulatoire de Schrödinger, une méthode générale de résolution par approximations successives, Compt. Rend., 183, 24-26, 1926.

Carpenter, D. L., et al., Small-scale field-aligned plasmaspheric density structures inferred from Radio Plasma Imager on IMAGE, J. Geophys. Res., 107(A9), 1258, doi:10.1029/2001JA009199, 2002.

Chang, T., Lower-hybrid collapse, caviton turbulence, and charged particle energization in the topside auroral ionosphere and magnetosphere, Phys. Fluids B, 5(7), 2646-2656, 1993.

Delory, G. T., Rocket observations of VLF waves, electron precipitation and ion heating in the auroral ionosphere, Ph.D. thesis, Univ. of Calif., Berkeley, 1996.

Delory, G., P. Schuck, C. Seyler, R. Ergun, C. Carlson, and J. McFadden, Measurements of auroral VLF bursts as rotating eigenmode structures on the alaska '93 sounding rocket, Eos Trans. $A G U$, 79(17), Spring Meet. Suppl., S315, 1998.

Doolen, G. D., D. F. DuBois, and H. A. Rose, Nucleation of cavitons in strong langmuir turbulence, Phys. Rev. Lett., 54(8), 804-807, 1985.

Dovner, P. O., A. I. Eriksson, R. Boström, and B. Holback, Freja multiprobe observations of electrostatic solitary structures, Geophys. Res. Lett., 21(27), 1827-1834, 1994.

Dovner, P. O., A. I. Eriksson, R. Boström, and B. Holback, The occurrence of lower hybrid cavities in the upper ionosphere, Geophys. Res. Lett., 24(5), 619-622, 1997.

Ergun, R. E., E. M. Klementis, G. T. Delory, C. W. Carlson, and J. P. McFadden, Plasma density observations during large-amplitude lower hybrid emissions, Eos Trans. $A G U, 74(44)$, Fall Meet. Suppl., F552, 1994.

Ergun, R. E., E. M. Klemetis, G. T. Delory, J. P. McFadden, and C. W. Carlson, VLF wave localization in the low-altitude auroral region, Geophys. Res. Lett., 22(16), 2099-2102, 1995. 
Eriksson, A. I., B. Holback, P. O. Dovner, R. Boström, G. Holmgren, M. André, L. Eliasson, and P. M. Kintner, Freja observations of correlated small-scale density depletions and enhanced lower hybrid waves, Geophys. Res. Lett., 21(27), 1843-1846, 1994.

Ghielmetti, A. G., R. G. Johnson, R. D. Sharp, and E. G. Shelley, The latitudinal, diurnal, and altitudinal distributions of upflowing energetic ions of ionospheric origin, Geophys. Res. Lett., 5(1), 59-62, 1978.

Gorney, D. J., A. Clarke, D. Croley, J. Fennell, J. Luhmann, and P. Mizera The distribution of ion beams and conics below $8000 \mathrm{~km}, J$. Geophys Res, 86, 83-89, 1981.

Horwitz, J. L., Conical distributions of low-energy ion fluxes at synchronous orbit, J. Geophys. Res., 85, 2057-2064, 1980.

Høymork, S. H., H. L. Pécseli, B. Lybekk, J. Trulsen, and A. Eriksson, Cavitation of lower hybrid waves in the Earth's ionosphere: A model analysis, J. Geophys. Res., 105, 18,519-18,535, 2000.

Høymork, S. H., H. L. Pecseli, B. Lybekk, J. Trulsen, and A. Eriksson, The shape and evolution of lower hybrid density cavities observed by FREJA, Phys. Chem. Earth, Part C, Sol. Terr. Planet. Sci., 26(1-3), 213-217, 2001.

Jeffreys, H., On certain approximate solutions of linear differential equations of the second order, Proc. London Math. Soc., Ser. 2, 23, 423-436, 1923.

Kintner, P. M., Plasma waves and transversely accelerated ions in the terrestrial ionosphere, Phys. Fluids B, 4(7), 2264-2269, 1992.

Kintner, P. M., J. Vago, S. Chesney, R. L. Arnoldy, K. A. Lynch, C. J. Pollock, and T. E. Moore, Localized lower hybrid acceleration of ionospheric plasma, Phys. Rev. Lett., 68(16), 2448-2451, 1992.

Kintner, P. M., P. W. Schuck, and J. Franz, Spaced measurements and progress in understanding space plasma waves, Phys. Plasmas, 7(5), $2135-2141,2000$

Kjus, S. H., H. L. Pecseli, B. Lybekk, J. Holtet, J. Trulsen, and A. Eriksson, Solitary waves in the Earth's upper ionosphere, in Solitons and Coherent Structures in Physics and Biology, SOLPHYS '97 Proceedings, Tech. Univ. of Denmark, Lyngby, 1997.

Kjus, S. H., H. L. Pécseli, B. Lybekk, J. Holtet, J. Trulsen, H. Lür, and A. Eriksson, Statistics of the lower hybrid wave cavities detected by the Freja satellite, J. Geophys. Res., 103, 26,633-26,647, 1998

Klumpar, D. M., Transversely accelerated ions: An ionospheric source of hot magnetospheric ions, J. Geophys. Res., 84, 4229-4237, 1979.

Knudsen, D. J., P. O. Dovner, A. I. Eriksson, and K. A. Lynch, The effect of lower hybrid cavities on core plasma observed by Freja, J. Geophys. Res. 103, 4241-4249, 1998

Knudsen, D. J., D. D. Wallis, and H. G. James, Tethered two-point measurements of solitary auroral density cavities, Geophys. Res. Lett., 26(19) 2933-2936, 1999

Knudsen, D. J., J. K. Burchill, D. D. Wallis, R. F. Pfaff, J. H. Clemmons, and S. R. Bounds, Electromagnetic wave and particle signatures of lowerhybrid cavities, Eos Trans. AGU, 82(20), Spring Meet. Suppl., S358, 2001.

Kramers, H. A., Wellenmechanik und halbzahlige quantisierung, Z. Physik, $39,828-840,1926$

Labelle, J., P. M. Kintner, A. W. Yau, and B. A. Whalen, Large amplitude wave packets observed in the ionosphere in association with transverse ion acceleration, J. Geophys. Res., 91, 7113-7118, 1986.

Lynch, K. A., R. L. Arnoldy, P. M. Kintner, and J. L. Vago, Electron distribution function behavior during localized transverse ion acceleration events in the topside auroral zone, J. Geophys. Res., 99, 2227-2241, 1994

Lynch, K. A., R. L. Arnoldy, P. M. Kintner, and J. Bonnell, The AMICIST auroral sounding rocket: A comparison of transverse ion acceleration mechanisms., Geophys. Res. Lett., 23(23), 3293-3296, 1996.

Lynch, K. A., R. L. Arnoldy, P. M. Kintner, P. Schuck, J. W. Bonnell, and V. Coffey, Auroral ion acceleration from lower hybrid solitary structures: A summary of sounding rocket observations, J. Geophys. Res., 104(A12), 28,515-28,534, 1999 .

McAdams, K. L., J. Labelle, P. W. Schuck, and P. M. Kintner, PHAZE II observations of lower hybrid burst structures occurring on density gradients, Geophys. Res. Lett., 25(16), 3091-3094, 1998.

Melatos, A., and P. A. Robinson, Properties of transit-time interactions in magnetized plasmas: Analytic and numerical results, Phys. Fluids. B, 5(8), 2751-2763, 1993.

Melatos, A., and P. A. Robinson, Local transit-time damping in a magnetic field, and the arrest of lower-hybrid wave collapse, Phys. Plasmas, 3(4), $1263-1279,1996$.
Mizera, P. F., and J. F. Fennell, Signatures of electric fields from high and low altitude particle distributions, Geophys. Res. Lett., 4(8), 311-314, 1977.

Pécseli, H. L., K. Iranpour, O. Holter, B. Lybekk, J. Holtet, J. Trulsen, A. Eriksson, and B. Holback, Lower-hybrid wave cavities detected by the Freja-satellite, J. Geophys. Res., 101, 5299, 1996

Pécseli, H. L., B. Lybekk, J. Trulsen, and A. Eriksson, Lower-hybrid wave cavities detected by instrumented spacecraft, Plasma Phys. Control. Fusion, 39(5A), 236-277, 1997.

Pinsçon, J.-L., P. M. Kintner, P. W. Schuck, and C. E. Seyler, Observation and analysis of lower hybrid solitary structures as rotating eigenmodes, J. Geophys. Res., 102, 17,283-17,296, 1997.

Robinson, P. A., Scalings, specta, and statistics of strong wave turbulence, Phys. Plasmas, 3(1), 192-201, 1996.

Robinson, P. A., Nonlinear lower hybrid solitary structures in auroral plasmas: Comparison of theory with observations, Adv. Space Res., 23(10), $1679-1688,1999$

Rosenberg, S., and W. Gekelman, Electric field measurements of directly converted lower hybrid waves at a density striation, Geophys. Res. Lett., 25(6), 865-868, 1998.

Rosenberg, S., and W. Gekelman, A laboratory investigation of lower hybrid wave interactions with a field-aligned density depletion, Geophys. Res. Lett., 27(6), 859-862, 2000

Rosenberg, S., and W. Gekelman, A three-dimensional study of lower hybrid wave interactions with field-aligned density depletions, J. Geophys. Res., 106, 28,867-28,884, 2001

Santolík, O., and D. A. Gurnett, Propagation of auroral hiss at high altitudes, Geophys. Res. Lett., 29(10), doi:10.1029/2001GL013666, 2002.

Schuck, P. W., A theory of lower hybrid solitary structures: Rotating helical eigenstates excited by wave scattering, Ph.D. thesis, Applied Physics, Cornell Univ., Ithaca, N.Y., May 1999.

Schuck, P. W., C. E. Seyler, J.-L. Pinçon, J. W. Bonnell, and P. M. Kintner, Theory simulation and observation of bound states associated with lower hybrid solitary structures, J. Geophys. Res., 103, 69356953, 1998.

Schuck, P. W., G. I. Ganguli, and P. M. Kintner, On the role of lower hybrid collapse in the auroral ionosphere, Phys. Rev. Lett., 89(6), 2002.

Seyler, C. E., Lower hybrid wave phenomena associated with density depletions, J. Geophys. Res., 99, 19,513-19,525, 1994

Shapiro, V. D., V. I. Shevchenko, G. I. Solov'ev, V. P. Kalinin, R. Bingham, R. Z. Sagdeev, M. Ashora-Abdalla, J. Dawson, and J. J. Su, Wave collapse at the lower hybrid resonance, Phys. Fluids B, 5(9), 3148-3162, 1993.

Shapiro, V. D., G. I. Soloviev, J. M. Dawson, and R. Bingham, Lower hybrid dissipative cavitons and ion heating in the auroral ionosphere, Phys. Plasmas, 2(2), 516-526, 1995.

Shelley, E. G., Heavy ions in the magnetosphere, Space Sci. Rev., 23(3), 465-497, 1979

Shelley, E. G., R. D. Sharp, and R. G. Johnson, Satellite observations of an ionospheric acceleration mechanism, Geophys. Res. Lett., 3(11), 654656,1976

Stenzel, R. L., and W. Gekelman, Electrostatic waves near the lower hybrid resonance, Phys. Rev. A, 11(6), 2057-2060, 1975.

Swansen, D. G. Plasma Waves, Academic, San Diego, Calif, 1989

Vago, J. L., P. M. Kintner, S. W. Chesney, R. L. Arnoldy, K. A. Lynch, T. E. Moore, and C. J. Pollock, Transverse ion acceleration by localized lower hybrid waves in the topside auroral ionosphere, J. Geophys. Res., 97, $16,935-16,957,1992$

Weinberg, S., Eikonal method in magnetohydrodynamics, Phys. Rev., 126(6), 1899-1909, 1962.

Wentzel, G., Eine verallgemeinerung der quantenbedingungen für die zwecke der wellenmechanik, Z. Physik, 38, 518-529, 1926.

Whalen, B. A., W. Bernstein, and D. W. Daly, Low-altitude acceleration of ionospheric ions, Geophys. Res. Lett., 5(1), 55-58, 1978.

J. W. Bonnell, Space Sciences Laboratory, University of California, Centennial Dr. at Grizzly Peak Blvd., Berkeley, CA 94720-7450, USA. (jbonnell@ssl.berkeley.edu)

P. W. Schuck, Plasma Physics Division, Naval Research Laboratory, Code 6794, 4555 Overlook Ave., SW, Washington, DC 20375-5000, USA (schuck@ppdmail.nrl.navy.mil) 